\title{
Alignment of Stellar and AGN Accretion Disks from SDSS Data
}

\section{Claudia del P. Lagos ${ }^{1}$, Nelson D. Padilla ${ }^{2}$, Michael A. Strauss ${ }^{3}$, and Lei $\mathrm{Hao}^{4}$}

${ }^{1}$ Institute for Computational Cosmology, Department of Physics, University of Durham, South Road, Durham DH1 3LE, UK

Email: c.d.p.lagos@durham.ac.uk

${ }^{2}$ Departamento Astronomía y Astrofísica, Universidad Católica de Chile, Av Vicuña Mackenna 4860, Santiago, Chile

${ }^{3}$ Department of Astrophysical Sciences, Princeton University, Princeton, NJ 08544, USA

${ }^{4}$ McDonald Observatory, University of Texas, Austin, TX 78712, USA

Keywords. galaxies: active, galaxies: elliptical, galaxies: spiral

Lagos, Padilla \& Cora (2009) show that if alignments between the galaxy kinematics and the AGN system were to occur, massive galaxies should host BHs with high spin values, regardless of the detailed physics of the BH. Since the BH spin regulates the mass-to-energy conversion (Marconi et al. 2004) and possibly the existence of radio jets (Sikora et al. 2007), this study has a strong impact in our understanding of galaxy formation.

We study the intrinsic shapes and orientations of type 1 and 2 AGN galaxies in the Sloan Digital Sky Survey Data Release 4 (SDSS DR4, described by Hao et al. 2005), by studying the distribution of projected axis ratios of AGN hosts separated into spiral and elliptical galaxies. We define control samples of non-AGN galaxies that mimic the color, luminosity, and concentration distributions of the AGN population, taking into account the effects of dust extinction and reddening. Assuming that AGN populations have the same underlying shapes as their corresponding control samples we find that the type 1 AGN population is strongly biased toward face-on galaxies, while the spiral type 2 AGN toward edge-on galaxies. Those tendencies could be explained with a central obscuring torus of $\sim 40^{\circ}$ of azimuthal height located preferentially in the galactic plane. This points towards a geometrical alignment between optical light and accretion disks (the Figure shows the distribution of $\cos \theta$ of AGN). The details of this work are described by Lagos, Padilla, Strauss, \& Hao (in preparation).
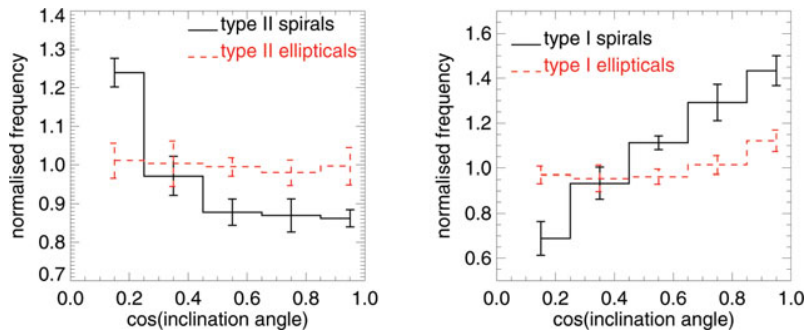

Figure 1. Distribution of inclination angles of type 2 (left) and type 1 (right) host AGN galaxies. Errors were calculated using the jackknife technique.

\section{References}

Hao, L., et al. 2005, AJ, 129, 1783

Lagos, C. P., Padilla, N. D., \& Cora S. A. 2009, MNRAS, 395, 625

Marconi, A., et al. 2004, MNRAS, 351, 169

Padilla, N. D. \& Strauss, M. A. 2008, MNRAS, 388, 1321

Sikora, M., Stawarz, L., \& Lasota, J. P. 2007, ApJ, 667, 704 